\title{
Epigenetic mechanism and target therapy of UHRFI protein complex in malignancies
}

REVIEW

This article was published in the following Dove Medical Press journal:

OncoTargets and Therapy

Busheng Xue,' Jiansong

Zhao,' Penghui Feng, ${ }^{2}$ Jia

Xing, ${ }^{3}$ Hongliang $\mathrm{Wu},{ }^{\prime}$

Yan $\mathrm{Li}^{\prime}$

'Department of Spine and Joint Surgery, Shengjing Hospital, China Medical University, Shenyang, People's Republic of China; ${ }^{2}$ Department of Obstetrics and GynecologyReproductive Medical Center, Shengjing Hospital, China Medical University, Shenyang, People's Republic of China; ${ }^{3}$ Department of Histology and Embryology, Basic Medicine College, China Medical University, Shenyang, People's Republic of China

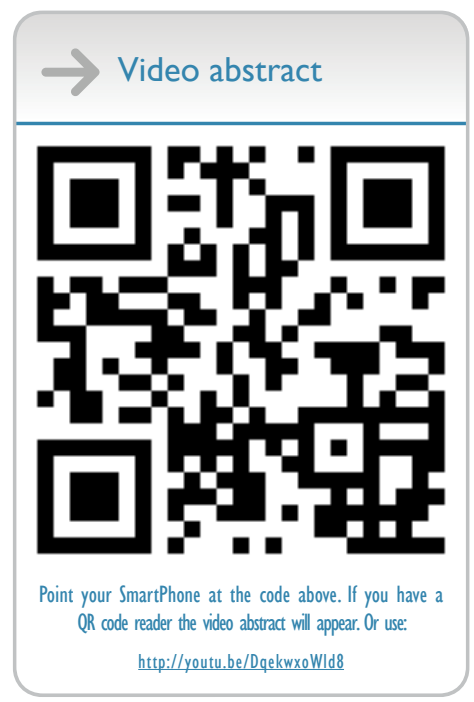

Correspondence: Yan Li

Department of Spine and Joint Surgery,

Shengjing Hospital, China Medical

University, No 36 Sanhao Street,

Heping District, Shenyang I 10004 ,

People's Republic of China

Tel +862496615 331II

Email liy15@sj-hospital.org

\begin{abstract}
Ubiquitin-like with plant homeodomain and really interesting new gene finger domains 1 (UHRF1) functions as an epigenetic regulator recruiting PCNA, DNMT1, histone deacetylase 1, G9a, SuV39H, herpes virus-associated ubiquitin-specific protease, and Tatinteractive protein by multiple corresponding domains of DNA and H3 to maintain DNA methylation and histone modifications. Overexpression of UHRF1 has been found as a potential biomarker in various cancers resulting in either DNA hypermethylation or global DNA hypomethylation, which participates in the occurrence, progression, and invasion of cancer. The role of UHRF1 in the reciprocal interaction between DNA methylation and histone modifications, the dynamic structural transformation of UHRF1 protein within epigenetic code replication machinery in epigenetic regulations, as well as modifications during cell cycle and chemotherapy targeting UHRF1 are evaluated in this study.
\end{abstract}

Keywords: UHRF1 protein complex, epigenetic modification, ICBP90

\section{Introduction}

Ubiquitin-like with plant homeodomain (PHD) and really interesting new gene (RING) finger domain 1 (UHRF1, also called ICBP90 or Np95) was identified as a transcription factor which could regulate the expression of topoisomerase II $\alpha$ by binding to an inverted CCAAT box located in its promoter. ${ }^{1}$ The nuclear protein UHRF1 consists of multiple domains such as ubiquitin-like domain (UBL), tandem tudor domain (TTD; TTD $^{\mathrm{N}}$ and $\mathrm{TTD}^{\mathrm{C}}$ ), PHD, SET and RING-associated domain (SRA) as well as RING domain, which play an important role in the epigenetic regulation of gene expression and tumorigenesis.

Epigenetic modification refers to inherited changes in gene expression without variations in DNA structure or sequence ${ }^{2-4}$ and includes DNA methylation, histone modification, genome imprinting, $\mathrm{X}$-chromosome inactivation, and microRNA regulation, ${ }^{4,5}$ where DNA methylation and histone modification are most important and their aberrant changes are always involved in cancer and neurological diseases. UHRF1 serves as a key regulator that participates in both DNA methylation and histone modifications.

\section{UHRFI allows crosstalk between DNA methylation and histone code as an epigenetic coordinator}

DNA methylation is a fundamental epigenetic process in the regulation of gene expression. The process occurs at the carbon-5 position of cytosines mainly within $\mathrm{CpG}$ sites mediated by DNA methyltransferases (DNMTs). In detail, DNMT3A and DNMT3B are mainly responsible for de novo methylation during gametogenesis and early embryonic 
development, whereas DNMT1 preferentially methylates hemi-methylated $\mathrm{CpG}$ sites for maintenance of DNA methylation by a self-inhibitory mechanism during DNA replication and cell division. ${ }^{6}$ Aberrant DNA methylation can lead to abnormal embryogenesis, ${ }^{7}$ neurological diseases, ${ }^{8}$ and cancers, ${ }^{9,10}$ including global DNA hypomethylation and specific $\mathrm{CpG}$ island hypermethylation in which the former can lead to chromosomal instability, ${ }^{11}$ activation of certain transcription factors, ${ }^{12}$ and loss of genetic imprints; ${ }^{13}$ the latter often results in the inhibition of tumor suppressor genes.

Eukaryotic DNMT1 is composed of an N-terminal nuclear localization sequence, a replication foci targeting sequence (RFTS) that localizes DNMT1 to the DNA replication fork, a zinc finger CXXC domain that specifically recognizes unmethylated $\mathrm{CpG}$ dinucleotides, ${ }^{14,15}$ a pair of bromo-adjacent homology (BAH) domains, and a C-terminal methyltransferase domain including the catalytic core and the target recognition domain (TRD). Although the occlusion of the CXXC-BAH1 linker at the catalytic site and restraint by BAH2-TRD loop preventing TRD from binding to unmethylated CpG sites facilitate DNMT1-mediated maintenance of DNA methylation, ${ }^{16,17}$ the auto-inhibitory role of RFTS domain and DNMT1-interacting proteins which affect its activity, such as UHRF1, have been revealed. ${ }^{18,19}$ UHRF1 recognizes and binds to hemi-methylated DNA (hmDNA) through its SRA domain by the thumb (444-499 residues) and NKR finger (483-496 residues) sub-domains targeting minor and major grooves, respectively, ${ }^{20}$ and recruits DNMT1 by targeting the RFTS domain which leaves the catalytic pocket of DNMT1 in the S phase of the cell cycle to replication foci ${ }^{21,22}$ and relieves the auto-inhibitory activity of DNMT1 to maintain the cytosine in newly synthesized DNA methylation with high fidelity. In addition, the UHRF1 C-terminal RING finger functions as an ubiquitin E3 ligase to establish histone H3 ubiquitination at Lys23 and Lys18 recognized by the RFTS domain of DNMT123,24 to promote its localization onto replication foci, which also has a prerequisite role in the maintenance of DNA methylation. Recently, the N-terminal UBL domain of UHRF1 was found to bind directly to DNMT1 enhancing DNMT1 enzymatic activity toward newly replicated chromatin by controlling targeted H3 ubiquitylation through a hydrophobic patch. ${ }^{25,26}$ Furthermore, the UBL domain can bind the E2 Ube2D and form a stable $\mathrm{E} 2 / \mathrm{E} 3 /$ chromatin complex that is equally required for the DNMT1-mediated maintenance of DNA methylation. ${ }^{26,27}$

Posttranslational modification of histone participates in DNA replication, DNA damage response, chromosome translocation, transcription activation and suppression,
X chromosome inactivation, and heterochromatin replication. ${ }^{28}$ To date, the most studied histone modifications include the methylation of arginine (R) and lysine (K), acetylation of lysine, phosphorylation of serine $(\mathrm{S})$ and threonine $(\mathrm{T})$, and lysine ubiquitination. The histone lysine methylation is divided into monomethylation, dimethylation, and trimethylation, which greatly increases the complexity of histone modification and gene expression. Lysine acetylation of histone tails leads to transcriptional activation by neutralization of a positive charge at a lysine side chain, triggering a detachment of the side chain from the negatively charged DNA strands.

Methylation of histone $\mathrm{H} 3$ plays an important role in transcription, among which $\mathrm{H} 3 \mathrm{~K} 4$ methylation often causes transcriptional activation while $\mathrm{H} 3 \mathrm{~K} 9$ methylation always coordinates gene silencing. Unmethylated or methylated lysine can be modified by some proteins to regulate gene expression. Generally, UHRF1 recognizes the dimethylated or trimethylated $\mathrm{H} 3 \mathrm{~K} 9$ (H3K9me2/3) mediated by $\mathrm{G} 9 \mathrm{a}^{29}$ or SuV39H through $\mathrm{TTD}^{\mathrm{N}}$ and PHD (TTD-PHD) as well as identifies unmodified arginine 2 (R2) and unmodified lysine 4 (K4) through the PHD domain. ${ }^{30-36}$ The association with histone marks are all required for DNA methylation. ${ }^{34,37}$ Moreover, UHRF1 reportedly adopts a closed conformation in the absence of chromatin in which a polybasic region (PBR) in the C-terminus binds to the TTD and inhibits its recognition of $\mathrm{H} 3 \mathrm{~K} 9 \mathrm{me} 3$, whereas the SRA domain binds to the PHD domain and inhibits recognition of unmethylated histone $\mathrm{H} 3$ at residue $\mathrm{R} 2$ (H3R2); upon binding to hmDNA by the SRA domain, UHRF1 impairs the intramolecular interactions and transfers to an open state, ${ }^{30,34,38}$ which allows TTD-PHD to recognize H3K9me3 and facilitates SRA-PBR to either recognize hmDNA or recruit DNMT1 for an accurate methylation heredity ${ }^{36}$ (Figure 1 ). In addition, phosphatidylinositol 5-phosphate (PI5P) in the nucleus can interact with the PBR through phosphorylation of S651 to participate in the regulation of heterochromatin localization of UHRF1 and crosstalk between H3K9 methylation and DNA methylation. ${ }^{39,40}$ Phosphorylation of S298 in the linker residue abrogates the UHRF1-H3 interaction by altering the relative position of the two reader modules, indicating the linker region may act as a functional switch of UHRF1 involved in multiple regulatory pathways such as maintenance of DNA methylation, transcriptional repression, and cell cycle progression. ${ }^{41}$ In contrast, Zhao et $\mathrm{al}^{42}$ found only a $10 \%$ reduction of DNA methylation in various tissues after abolishment of the association between $\mathrm{H} 3 \mathrm{~K} 9 \mathrm{me} 2 / 3$ and UHRF1 in mammals, indicating that $\mathrm{H} 3 \mathrm{~K} 9$ methylation binding of UHRF1 is not the only process to ensure 


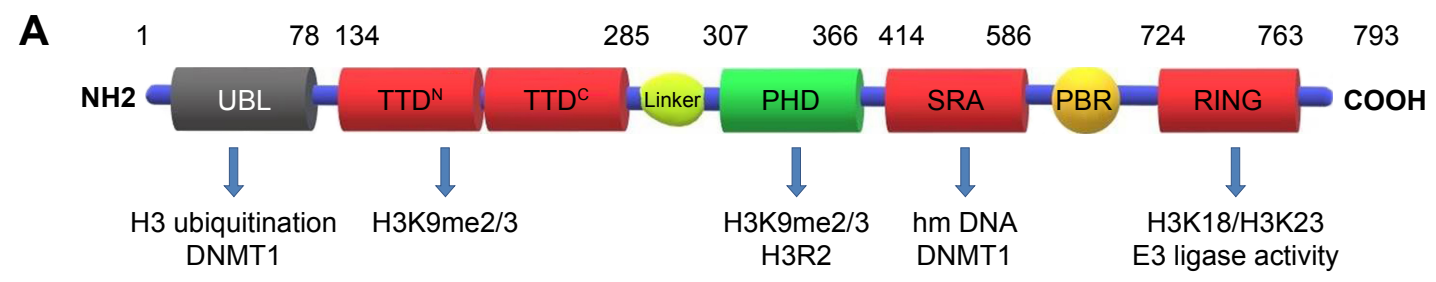

B
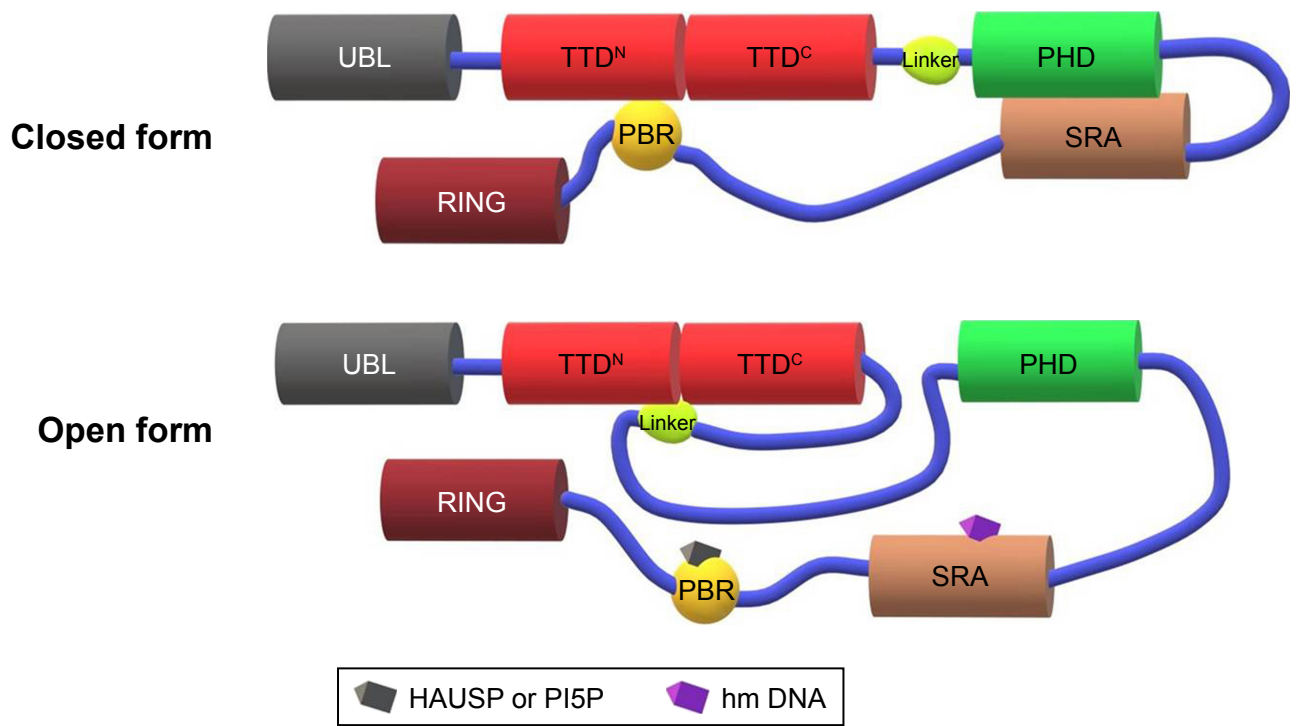

Figure I Schematic representation of UHRFI with structure-function domains in the maintenance of DNA methylation.

Notes: (A) Domain architecture of UHRFI with their corresponding epigenetic function including DNA methylation and histone modification. (B) Conformational changes of UHRFI from close to open state mediated by hmDNA.

Abbreviations: DNMT, DNA methyltransferases; H3K9me2/3, dimethylated or trimethylated H3K9; H3R2, unmethylated histone H3 at residue R2; hmDNA, hemimethylated DNA; HAUSP, herpes virus-associated ubiquitin-specific protease; PBR, polybasic region; PHD, plant homeodomain; PI5P, phosphatidylinositol 5-phosphate; RING, really interesting new gene; SRA, SET and RING-associated domain; TTD, tandem tudor domain; UBL, ubiquitin-like domain.

DNA methylation. A positive correlation exists between UHRF1 and EZH2 in cancer cells, ${ }^{43,44}$ and EZH2 participates in H3K27 methylation which mediates gene silencing. ${ }^{45,46}$ Ferry et $\mathrm{al}^{47}$ found nonhistone mammalian DNA ligase 1 (LIG1), which contains a conserved H3K9-like mimic can also be methylated by G9a/GLP at K126 and subsequently recruit UHRF1 to replication foci, which is similar to binding H3K9me2/3 to maintain DNA methylation.

UHRF1 can also lead to global DNA hypomethylation in cancers. ${ }^{4-50}$ Long interspersed nucleotide element-1 (LINE1) is considered a surrogate marker of global DNA methylation. ${ }^{51}$ Nakamura et $\mathrm{a}^{48}$ found that overexpression of UHRF1 could drive global DNA hypomethylation in esophageal squamous cell carcinoma (ESCC). Paradoxically, Ye et $\mathrm{al}^{52}$ reported that knockdown of UHRF1 results in LINE1 hypomethylation in ESCC. Because the states of promoter methylation in tumor suppressor genes were not mentioned in these two studies, Hoshimoto et $\mathrm{al}^{53}$ hypothesized that ESCC was associated with global DNA hypomethylation and specific tumor-related gene hypermethylation.
Although DNMT1 has a 30- to 40-fold preference for hmDNA sites, ${ }^{54}$ it can still bind to unmethylated DNA, and exert de novo methylation activity. ${ }^{55,56}$ UHRF1 may lead to aberrant DNA methylation state in cancer cells for two reasons: 1) UHRF1 recruits DNMT1 to $\mathrm{hm}-\mathrm{CpG}$ islands or CpG islands, which have not been previously methylated because DNMT1 also has the property of de novo methylation ${ }^{19,56}$ which undergoes hypermethylation, over time, finally leading to the malignancy of cancer; 2) when the UHRF1 protein levels become high, ubiquitination may occur, and DNMT1 is ubiquitylated to a proper level to maintain the malignancy state of cancer, including hypermethylation of specific tumor suppressor gene promoters as well as global DNA hypomethylation.

\section{Self-regulation of UHRF I macromolecular complex during the cell cycle}

UHRF1, DNMT1, HAUSP, HDAC1, Tip60, Hsp90, Suv39H1, PCNA, and pRb form a macromolecular complex 
termed epigenetic code replication machinery (ECREM) ${ }^{57}$ to undergo temporal and spatial control during the cell cycle. During DNA replication, DNMT1 is partially recruited into the replication forks by PCNA. ${ }^{58-60}$ Tip60 interacts with SRA and RING domain of UHRF $1^{61}$ through its enzymatic MYST domain, and overexpression of Tip60 leads to the downregulation of UHRF $1 .{ }^{62}$ In addition, UHRF1 can repress the activity and expression of Tip60. ${ }^{63}$ Furthermore, overexpression of Tip60 leads to acetylation and subsequent ubiquitylationdependent proteasomal degradation of DNMT1 mediated by UHRF1. ${ }^{64}$ Although increased DNMT1 abundance in multiple cancers is largely due to the reduced degradation at the protein level rather than higher mRNA level, ${ }^{65,66}$ the consequence appears diverse based on cell cycle status or cell types, ${ }^{63,67-69}$ and DNMT1 stability might be regulated by other upstream factors such as pRb and ATM. ${ }^{70}$ However, the mechanism remains to be elucidated.

Conversely, HAUSP interacts with PBR of UHRF1 through UBL1 and UBL2 domains to stabilize UHRF1 via deubiquitination and protects it from autoubiquitination as well as promote the open state of UHRF1 to facilitate the binding of UHRF1 to H3K9me3. ${ }^{34,71-73}$ When S652 (located in PBR) is phosphorylated by $\mathrm{M}$ phase-specific kinase CDK1-cyclin $\mathrm{B}^{71}$ during mitosis, UHRF1 is separated from HAUSP and the PBR domain is exposed to Tip60 for acetylation and ultimate degradation. ${ }^{39}$ In addition, HAUSP can deubiquitinate Tip60. ${ }^{74}$

HAUSP binds to the KG linker of DNMT1 at its acidic pocket near the $\mathrm{C}$-terminal to deubiquitinate DNMT1 from proteasomal degradation. ${ }^{64}$ In addition, HAUSP functions as a deubiquitylating enzyme toward ubiquitylated histone $\mathrm{H} 3$, and is likely involved in DNMT1 recruitment to DNA replication sites and the regulation of maintenance of DNA methylation without affecting the kinetics and efficiency of DNA replication. ${ }^{75}$ Simultaneously, Cheng et al $^{76}$ found that acetylation of the KG linker lysine residues impairs DNMT1-HAUSP interaction and promotes the degradation of DNMT1. Treatment with HDAC inhibitor not only increases the level of acetylated DNMT1 but also decreases the total DNMT1 protein level. HDACs can be recruited by both DNMT1 and UHRF1 to repress gene expression, and HDAC1 can deacetylate DNMT1 to protect it from degradation. ${ }^{6477}$ In addition, the stability of DNMT1 is maintained in part through the recruitment of Hsp90 mediated by the ubiquitin-proteasome pathway. ${ }^{78}$ Suv39H1 and G9a are both H3K9 histone methyltransferases found in the same macromolecular complex with $\mathrm{UHRF} 1^{43,67,79}$ to prevent transcriptional activation; ${ }^{80,81}$ the latter can maintain the DNA methylation state required for de novo DNA methylation and the establishment in mouse embryonic stem cells. ${ }^{82-84}$ UHRF1 is necessary for binding Suv39H1 and H3K9me3 modification, ${ }^{43}$ however, the underlying interaction mechanisms remain to be elucidated.

The macromolecular complex may function as follows (Figure 2): UHRF1 and DNMT1 adopt a closed conformation and an auto-inhibitory state after protein synthesis, respectively. During DNA replication, the ECREM binds to replication foci. First, the closed conformation of UHRF1 shifts to an open state due to the following reasons: 1) HAUSP binds to PBR as well as the hmDNA; 2) SRA domain binds to hmDNA; and 3) phosphorylation of S651 by PI5P leads to TTD relief from PBR and forms a histone binding cassette with the PHD domain and linker. Second, UHRF1 in the open state binds to H3K9me $2 / 3$ or H3K9me3e mimic LIG1 by TTD or TTD-PHD as well as binds to H3R2 via the PHD domain. Third, the RING domain of UHRF1 ubiquitinates $\mathrm{H} 3 \mathrm{~K} 18$ and/or $\mathrm{H} 3 \mathrm{~K} 23$, which recruits the RFTS domain of DNMT1. The RFTS domain of DNMT1 also binds to the SRA domain or UBL of UHRF1 to relieve the self-inhibitory activity. HDAC1 and HAUSP can prevent UHRF1 and DNMT1 from acetylation and ubiquitination for degradation mediated by Tip60 during S phase when the abundance of Tip60 is relatively low. HAUSP can also deubiquitinate $\mathrm{H} 3$ which recruits the RFTS domain of DNMT1 to maintain a faithful inheritance of DNA methylation. At the beginning of G2 phase, when DNA replication is completed and methylation inheritance is formed, UHRF1 and DNMT1 undergo degradation. In detail, HAUSP dislocates from the UHRF1/DNMT1 complex due to phosphorylation of the S652 position by CDK1-cyclin B followed by ubiquitination of UHRF1. Degradation of UHRF1 leads to upregulation of Tip60 and acetylation of DNMT1, which is subsequently ubiquitylated by UHRF1 and other ubiquitinrelated enzymes. Finally, the abundance of UHRF1 and DNMT1 decreases to ensure the normal cell cycle.

\section{UHRF I regulation and target therapy}

Due to overexpression in various types of cancers such as gastric cancer, ${ }^{85}$ colorectal cancer, ${ }^{86}$ hepatocellular carcinoma, ${ }^{87}$ ESCC, ${ }^{48,88}$ prostate cancer, ${ }^{89,90}$ bladder cancer, ${ }^{91}$ breast cancer, ${ }^{92}$ lung cancer, ${ }^{93,94}$ melanoma, ${ }^{95}$ medulloblastoma, ${ }^{96}$ lymphoblastic leukemia, ${ }^{97}$ and osteosarcoma. ${ }^{98}$ UHRF1 appears a potential biomarker and is promising in the target therapy of cancers. High expression of UHRF1 inhibits a variety of tumor suppressor genes (Table 1) such as p16 $6^{I N K 4},{ }^{99-101}$ BRCA1, ${ }^{102}$ 


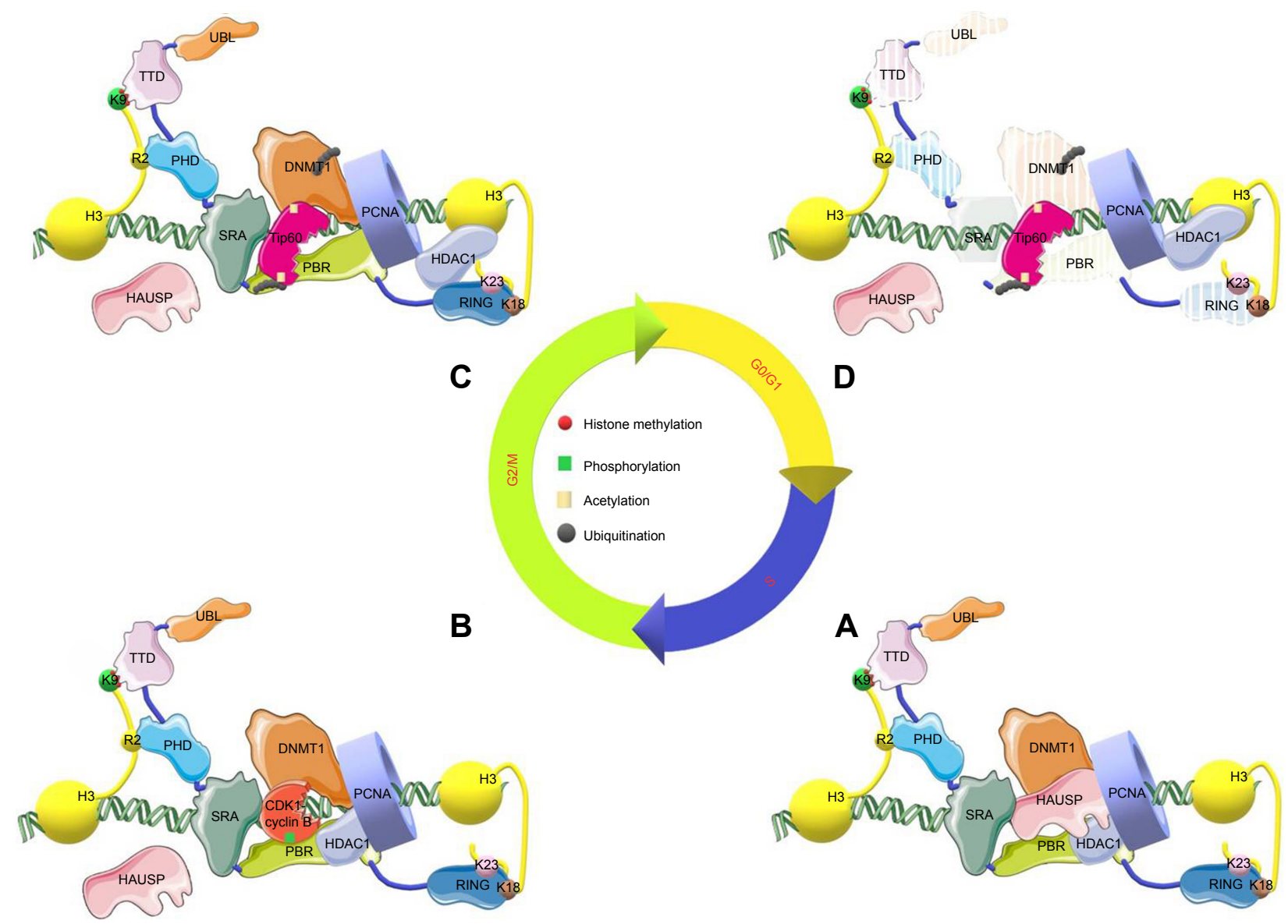

Figure 2 Working model for dynamic regulation of UHRFI macromolecular complex during cell cycle.

Notes: (A) The spatialization of UHRFI macromolecular complex including UHRFI at open state and other members of ECREM recruited into replication forks during $S$ phase. (B) The separation of HAUSP from the complex owing to phosphorylation mediated by CDKI-cyclin B at the beginning of G2 phase. (C) Acetylation and ubiquitination of UHRFI and DNMTI by Tip60 due to release of HAUSP and HDACI from ECREM during G2-M phase. (D) The ubiquitination-mediated protein degradation of UHRFI and DNMTI in order to enter the normal cell cycle.

Abbreviations: DNMT, DNA methyltransferases; ECREM, epigenetic code replication machinery; HAUSP, herpes virus-associated ubiquitin-specific protease; HDACI, histone deacetylase I; PBR, polybasic region; PHD, plant homeodomain; RING, really interesting new gene; SRA, SET and RING-associated domain; Tip60, Tat-interactive protein; TTD, tandem tudor domain; UBL, ubiquitin-like domain; UHRFI, ubiquitin-like with PHD and RING finger domains I; PCNA, proliferating cell nuclear antigen; CDKI, cyclin-dependent kinase I.

KISS1 $1{ }^{103} \mathrm{RASSF} 1,{ }^{94} \mathrm{MEG} 3,{ }^{104} \mathrm{CDH} 4,{ }^{85,105} \mathrm{Keapl},{ }^{106} \mathrm{KLF} 17,{ }^{107}$ RIP3, ${ }^{108}$ SHP1, ${ }^{109}$ RUNX3, FOXO4, CDX2, ${ }^{85}$ PPARG, ${ }^{110}$ Slit3 $,{ }^{105} C D H 1,{ }^{43,98,111}$ GPX3, ${ }^{43}$ IGFBP $3,{ }^{43,112}$ RGS $2,{ }^{113}$ $U B E 2 L 6,{ }^{114}$ and $m i R-145 .{ }^{115}$ Knowledge regarding the regulation of UHRF1 is limited; overexpression of UHRF1 may be involved in aberrantly high expression of transcription factors. Moreover, cell cycle regulators such as E2F8, E2F1, ${ }^{116,117}$ FOXM1,${ }^{118}$ leptin, SP1, ${ }^{119,120} \mathrm{Hsp} 90,{ }^{121} \mathrm{P} 53 / \mathrm{P} 21,{ }^{122}$ and WDR79 ${ }^{123}$ can also protect UHRF1 from ubiquitination. Conversely, microRNAs such as miR-124, ${ }^{124}$ microRNA-9, ${ }^{86}$ $m i R-101,{ }^{125} \mathrm{miR}-378, \mathrm{miR}-193 a-3 p,{ }^{126} \mathrm{miR}-1455 \mathrm{p} / \mathrm{miR}-145-3$ $p,{ }^{127} \mathrm{miR}-146 a / b,{ }^{105}$ and let-7a-3 ${ }^{128}$ play an important role in the downregulation of UHRF1 (Table 2). Most of the related microRNAs have been found suppressed in multiple cancers and can regulate the expression of UHRF1 by base pairing with 3' UTR at the mRNA level to inhibit protein synthesis.
The expression and activity of UHRF1 are also regulated by posttranslational modification. Chen et $\mathrm{al}^{129}$ found that casein kinase 1 delta could catalyze the phosphorylation of UHRF1 at S108 to undergo ubiquitination and degradation mediated by $\mathrm{SCF}^{\beta-\operatorname{TrCP}}$ in response to DNA damage; kinase Pim1 destabilizes UHRF1 by phosphorylation at S311; ${ }^{130}$ methyltransferase PRMT6 can induce H3R2me2a and inhibit the association of the PHD of UHRF1 with H3R2 leading to DNA hypomethylation, ${ }^{131}$ which provides new insights for cancer therapy targeting UHRF1. Graf et al ${ }^{132}$ found the LRR domain of Pramel7 in embryos interacts with the SRA and RING domain of UHRF1 which subsequently undergoes degradation and hypomethylation. Wang et $\mathrm{al}^{120}$ found that globular adiponectin inhibited leptin-stimulated cell proliferation in esophageal adenocarcinoma via the inhibition of UHRF1 mediated by adiponectin receptor 2 . 
Table I Inhibition of TSGs by overexpression of UHRFI in various types of cancers

\begin{tabular}{|c|c|c|}
\hline TSGs & Functions & Cancers \\
\hline $\mathrm{PI} 6^{\mathrm{INK} 4}$ & Growth, metastasis, and apoptosis & $\begin{array}{l}\text { Colorectal cancer }[22219067] \\
\text { Cervical cancer }[23688286] \\
\text { Glioblastoma }[25550546]\end{array}$ \\
\hline BRCAI & $\begin{array}{l}\text { DNA damage repair, transcription regulation, chromatin remodeling, cell cycle } \\
\text { checkpoint control, and apoptosis }\end{array}$ & Breast cancer [ 19943104$]$ \\
\hline RUNX3 & Hypoxia and immune cell maintenance & Hepatocellular carcinoma [26/47747] \\
\hline KISSI & $\begin{array}{l}\text { Tumor differentiation, the depth of invasion, lymph node metastasis, and } \\
\text { distant metastasis }\end{array}$ & Bladder cancer [252720I0] \\
\hline RASSFI & Proliferation, invasion, and apoptosis & Non-small-cell lung cancer [2135I083] \\
\hline MEG3 & Proliferation & Hepatocellular carcinoma [2564II94] \\
\hline $\mathrm{CDH} 4$ & Proliferation, invasion, and metastasis & Gastric cancer [26 |47747] [23982 |43] \\
\hline Keapl & Oxidative stress and reductive stress & Pancreatic cancer [26497II7] \\
\hline KLFI7 & Invasion and epithelial-mesenchymal transition & Breast cancer [28744404] \\
\hline SHPI & Tumor differentiation or muscular infiltration depth & Endometrial carcinoma [2659746I] \\
\hline $\mathrm{CD} \times 2$ & Lymph node metastasis and tumor, nodes, metastasis stage & Gastric cancer [26|47747] \\
\hline PPARG & Proliferation and migration and $\mathrm{Wnt} / \beta$-catenin signaling pathway & $\begin{array}{l}\text { Gastric cancer [26147747] } \\
\text { Colorectal cancer [22286757] }\end{array}$ \\
\hline FOXO4 & Proliferation and metastasis & Gastric cancer [26|47747] \\
\hline RIP3 & Cell survival, cell apoptosis, and cell necrosis & Colorectal cancer $[28981102]$ \\
\hline Slit3 & Invasion and metastasis & Gastric cancer [23982 |43] \\
\hline $\mathrm{CDHI}$ & Invasion and epithelial-to-mesenchymal transition & $\begin{array}{l}\text { Prostate cancer [22330I38] [29466696] } \\
\text { Osteosarcoma [26548607] }\end{array}$ \\
\hline IGFBP3 & Colony formation, migration, and invasion & $\begin{array}{l}\text { Prostate cancer }[22330138] \\
\text { Hepatoblastoma }[29507645]\end{array}$ \\
\hline GPX3 & ROS, migration, invasion, metastasis & Prostate cancer [22330I38] \\
\hline UBE2L6 & Apoptosis & Cervical cancer [29157076] \\
\hline RGS2 & Proliferation & Bladder cancer [25323766] \\
\hline miR-145 & Proliferation, migration and invasion & Non-small-cell lung cancer [2596/369] \\
\hline
\end{tabular}

Abbreviations: UHRFI, ubiquitin-like with PHD and RING finger domains I; TSGs, tumor suppressor genes; ROS, reactive oxygen species.

Anticancer drugs related to UHRF1 are as follows: uracil derivative NSC232003 ${ }^{133}$ functions as the only direct inhibitor by targeting fit within the SRA of UHRF1. Shikonin ${ }^{134}$ induces downregulation of both UHRF1 and DNMT1 in MDF-7 and hela cell lines. In addition, hinokitiol, ${ }^{68}$ dihydroartemisinin,${ }^{89}$ epigallocatechin-3-gallate, ${ }^{135}$ emodin, ${ }^{136}$ mTOR inhibitor torin-2, ${ }^{137}$ luteolin, ${ }^{69}$ ERK1/2 pathway inhibitor PD98059, LY294002, AG490, ${ }^{138}$ Hsp90 inhibitor 17-AAG or 17-dimethylamino- ethylamino-17-demethoxygeldanamycin, ${ }^{121}$ anisomycin, ${ }^{139}$ and curcumin ${ }^{95,140}$ have been reported to be used in various types of cancers.

Other functions of UHRF1 apart from epigenetic modification include its participation in the oxidative stress and DNA damage response ${ }^{141,142}$ to inhibit caspase-dependent apoptosis to promote the proliferation, invasion, and metastasis, ${ }^{143}$ promoting the development of embryogenesis and preimplantation of embryos, ${ }^{73,144}$ and regulating the

Table 2 Regulation of UHRFI by microRNA in various types of cancers

\begin{tabular}{l|l|l}
\hline mRNAs & Functions & Cancers \\
\hline miR-I24 & Proliferation, motility, angiogenesis & Bladder cancer [263I039I] \\
miR-9 & Proliferation, apoptosis & Colorectal cancer [25940709] \\
miR-10I & Migration, invasion & Renal cell cancer [27487I38] \\
miR-378 & Proliferation, apoptosis & Medulloblastoma [2890I497] \\
miR-193a-3p & Metastasis & Non-small-cell lung cancer [25833338] \\
miR-I45-5 p/miR-I45-3 P & Proliferation, apoptosis, migration, invasion & Bladder cancer [27628846] \\
miR-146a/b & Metastasis & Gastric cancer [23982I43] \\
& & Renal cell carcinoma [26859I4I] \\
\hline
\end{tabular}

Abbreviation: UHRFI, ubiquitin-like with PHD and RING finger domains I. 
function and development of T lymphocytes ${ }^{145,146}$ as well as the plasticity of smooth muscle cells. ${ }^{147}$

\section{Prospects}

Great progress has been made in the functional and modulation mechanisms of the UHRF1 protein complex, and it will likely become a universal biomarker for cancer and specific targets for cancer therapy. Emerging evidence indicates that UHRF1 modules do not act independently of each other but establish complex modes of interaction with patterns of chromatin modifications. Due to the variant abundance of ECREM and the distinct level of components in diverse cell lines, the UHRF1 protein complex possibly undergoes temporal and spatial control during the cell cycle, although the mechanisms remain to be elucidated. Furthermore, the phosphorylation sites of amino acids among the five domains are involved in the regulation of UHRF1 activity, protein stability, DNA methylation, and histone posttranscriptional modification. As mentioned above, the mechanisms regarding degradation of UHRF1 by acetylation and ubiquitination and specific drugs targeting UHRF1 need to be further clarified. Because many drugs can downregulate UHRF1 as well as DNMT1, and lower expression of DNMT1 is more sensitive to 5 -aza-CdR treatment, ${ }^{148}$ chemotherapy in cancer cells by using a DNMT1 inhibitor accompanied by UHRF1 inhibitor for the treatment of cancer is yet to be investigated. In summary, DNA methylation within $\mathrm{CpG}$ varies in different organs and times ${ }^{149}$ when cells are supposed to differentiate by epigenetic regulation, without DNA mutations or chromosomal translocation, indicating the important dynamics of UHRF1 in epigenetics and the various roles it plays over the life span.

\section{Acknowledgments}

This study was supported by National Natural Science Foundation of China (No 81272946) and Natural Science Foundation of Liaoning Province (No 2015020503 and 2015020473).

\section{Disclosure}

The authors report no conflicts of interest in this work.

\section{References}

1. Hopfner R, Mousli M, Jeltsch JM, et al. ICBP90, a novel human CCAAT binding protein, involved in the regulation of topoisomerase IIalpha expression. Cancer Res. 2000;60(1):121-128.

2. Levenson JM, Sweatt JD. Epigenetic mechanisms in memory formation. Nat Rev Neurosci. 2005;6(2):108-118.

3. Martin C, Zhang Y. Mechanisms of epigenetic inheritance. Curr Opin Cell Biol. 2007;19(3):266-272.
4. Probst AV, Dunleavy E, Almouzni G. Epigenetic inheritance during the cell cycle. Nat Rev Mol Cell Biol. 2009;10(3):192-206.

5. la Rosa AH, Manoharan M, Goolam AS. Current concepts of epigenetics in testicular cancer. Indian J Surg Oncol. 2017;8(2):169-174.

6. Gao L, Tan XF, Zhang S, et al. An intramolecular interaction of UHRF1 reveals dual control for its histone association. Structure. 2018;26(2): 304-311.

7. Jähner D, Stuhlmann H, Stewart CL, et al. De novo methylation and expression of retroviral genomes during mouse embryogenesis. Nature. 1982;298(5875):623-628.

8. Klein CJ, Botuyan M-V, Wu Y, et al. Mutations in DNMT1 cause hereditary sensory neuropathy with dementia and hearing loss. Nature Genetics. 2011;43(6):595-600.

9. Yang L, Rodriguez B, Mayle A, et al. DNMT3A loss drives enhancer hypomethylation in FLT3-ITD-associated leukemias. Cancer Cell. 2016;29(6):922-934.

10. Al-Kharashi LA, Al-Mohanna FH, Tulbah A, Aboussekhra A. The DNA methyl-transferase protein DNMT1 enhances tumor-promoting properties of breast stromal fibroblasts. Oncotarget. 2018;9(2): 2329-2343.

11. Yang AS, Estecio MR, Garcia-Manero G, Kantarjian HM, Issa JP. Comment on "chromosomal instability and tumors promoted by DNA hypomethylation" and "induction of tumors in mice by genomic hypomethylation”. Science. 2003;302(5648):1153.

12. Ogishima T, Shiina H, Breault JE, et al. Promoter CpG hypomethylation and transcription factor EGR1 hyperactivate heparanase expression in bladder cancer. Oncogene. 2005;24(45):6765-6772.

13. Tian F, Tang Z, Song G, et al. Loss of imprinting of IGF2 correlates with hypomethylation of the $\mathrm{H} 19$ differentially methylated region in the tumor tissue of colorectal cancer patients. Mol Med Rep. 2012;5(6): $1536-1540$.

14. Lee JH, Voo KS, Skalnik DG. Identification and characterization of the DNA binding domain of CpG-binding protein. J Biol Chem. 2001; 276(48):44669-44676.

15. Pradhan M, Estève PO, Chin HG, Samaranayke M, Kim GD, Pradhan S. CXXC domain of human DNMT1 is essential for enzymatic activity. Biochemistry. 2008;47(38):10000-10009.

16. Song J, Rechkoblit O, Bestor TH, Patel DJ. Structure of DNMT1-DNA complex reveals a role for autoinhibition in maintenance DNA methylation. Science. 2011;331(6020):1036-1040.

17. Song J, Teplova M, Ishibe-Murakami S, Patel DJ. Structure-based mechanistic insights into DNMT1-mediated maintenance DNA methylation. Science. 2012;335(6069):709-712.

18. Takeshita K, Suetake I, Yamashita E, et al. Structural insight into maintenance methylation by mouse DNA methyltransferase 1 (Dnmt1). Proc Natl Acad Sci U S A. 2011;108(22):9055-9059.

19. Bashtrykov P, Jankevicius G, Jurkowska RZ, Ragozin S, Jeltsch A. The UHRF1 protein stimulates the activity and specificity of the maintenance DNA methyltransferase DNMT1 by an allosteric mechanism. $J$ Biol Chem. 2014;289(7):4106-4115.

20. Avvakumov GV, Walker JR, Xue S, et al. Structural basis for recognition of hemi-methylated DNA by the SRA domain of human UHRF1. Nature. 2008;455(7214):822-825.

21. Bostick M, Kim JK, Estève PO, Clark A, Pradhan S, Jacobsen SE. UHRF1 plays a role in maintaining DNA methylation in mammalian cells. Science. 2007;317(5845):1760-1764.

22. Berkyurek AC, Suetake I, Arita K, et al. The DNA methyltransferase Dnmt1 directly interacts with the SET and RING finger-associated (SRA) domain of the multifunctional protein Uhrfl to facilitate accession of the catalytic center to hemi-methylated DNA. J Biol Chem. 2014;289(1):379-386.

23. Nishiyama A, Yamaguchi L, Sharif J, et al. Uhrf1-dependent H3K23 ubiquitylation couples maintenance DNA methylation and replication. Nature. 2013;502(7470):249-253.

24. Qin W, Wolf P, Liu N, et al. DNA methylation requires a DNMT1 ubiquitin interacting motif (UIM) and histone ubiquitination. Cell Res. 2015;25(8):911-929. 
25. Li T, Wang L, Du Y, et al. Structural and mechanistic insights into UHRF1-mediated DNMT1 activation in the maintenance DNA methylation. Nucleic Acids Res. 2018;46(6):3218-3231.

26. Foster BM, Stolz P, Mulholland CB, et al. Critical role of the UBL domain in stimulating the E3 ubiquitin ligase activity of UHRF1 toward chromatin. Mol Cell. 2018;72(4):739-752.

27. Darosa PA, Harrison JS, Zelter A, et al. A bifunctional role for the UHRF1 UBL domain in the control of hemi-methylated DNA-dependent histone ubiquitylation. Mol Cell. 2018;72(4):753-765.

28. Muratani M, Tansey WP. How the ubiquitin-proteasome system controls transcription. Nat Rev Mol Cell Biol. 2003;4(3):192-201.

29. Wozniak RJ, Klimecki WT, Lau SS, Feinstein Y, Futscher BW. 5-Aza2'-deoxycytidine-mediated reductions in G9A histone methyltransferase and histone $\mathrm{H} 3 \mathrm{~K} 9$ di-methylation levels are linked to tumor suppressor gene reactivation. Oncogene. 2007;26(1):77-90.

30. Cheng J, Yang Y, Fang J, et al. Structural insight into coordinated recognition of trimethylated histone $\mathrm{H} 3$ lysine 9 (H3K9me3) by the plant homeodomain (PHD) and tandem tudor domain (TTD) of UHRF1 (ubiquitin-like, containing PHD and RING finger domains, 1) protein. J Biol Chem. 2013;288(2):1329-1339.

31. Hu L, Li Z, Wang P, Lin Y, Xu Y. Crystal structure of PHD domain of UHRF1 and insights into recognition of unmodified histone $\mathrm{H} 3$ arginine residue 2. Cell Res. 2011;21(9):1374-1378.

32. Rajakumara E, Wang Z, Ma H, et al. PHD finger recognition of unmodified histone H3R2 links UHRF1 to regulation of euchromatic gene expression. Mol Cell. 2011;43(2):275-284.

33. Wang C, Shen J, Yang Z, et al. Structural basis for site-specific reading of unmodified R2 of histone $\mathrm{H} 3$ tail by UHRF1 PHD finger. Cell Res. 2011;21(9):1379-1382.

34. Rothbart SB, Dickson BM, Ong MS, et al. Multivalent histone engagement by the linked tandem Tudor and PHD domains of UHRF1 is required for the epigenetic inheritance of DNA methylation. Genes Dev. 2013;27(11):1288-1298.

35. Lallous N, Legrand P, McEwen AG, Ramón-Maiques S, Samama JP, Birck C. The PHD finger of human UHRF1 reveals a new subgroup of unmethylated histone $\mathrm{H} 3$ tail readers. PLoS One. 2011;6(11):e27599.

36. Fang J, Cheng J, Wang J, et al. Hemi-methylated DNA opens a closed conformation of UHRF1 to facilitate its histone recognition. Nat Commun. 2016;7(1):11197.

37. Rothbart SB, Krajewski K, Nady N, et al. Association of UHRF1 with methylated H3K9 directs the maintenance of DNA methylation. Nat Struct Mol Biol. 2012;19(11):1155-1160.

38. Arita $\mathrm{K}$, Isogai $\mathrm{S}$, Oda $\mathrm{T}$, et al. Recognition of modification status on a histone $\mathrm{H} 3$ tail by linked histone reader modules of the epigenetic regulator UHRF1. Proc Natl Acad Sci U S A. 2012;109(32): $12950-12955$.

39. Gelato KA, Tauber M, Ong MS, et al. Accessibility of different histone H3-binding domains of UHRF1 is allosterically regulated by phosphatidylinositol 5-phosphate. Mol Cell. 2014;54(6):905-919.

40. Rigbolt KT, Prokhorova TA, Akimov V, et al. System-wide temporal characterization of the proteome and phosphoproteome of human embryonic stem cell differentiation. Sci Signal. 2011;4(164):rs3.

41. Unoki M, Brunet J, Mousli M. Drug discovery targeting epigenetic codes: the great potential of UHRF1, which links DNA methylation and histone modifications, as a drug target in cancers and toxoplasmosis. Biochem Pharmacol. 2009;78(10):1279-1288.

42. Zhao Q, Zhang J, Chen R, et al. Dissecting the precise role of H3K9 methylation in crosstalk with DNA maintenance methylation in mammals. Nat Commun. 2016;7:12464.

43. Babbio F, Pistore C, Curti L, et al. The SRA protein UHRF1 promotes epigenetic crosstalks and is involved in prostate cancer progression. Oncogene. 2012;31(46):4878-4887.

44. Spodick DH. ECG diagnosis of MI in LBBB. Am Heart J. 1989; 117(6):1409.

45. McCabe MT, Ott HM, Ganji G, et al. EZH2 inhibition as a therapeutic strategy for lymphoma with EZH2-activating mutations. Nature. 2012; 492(7427):108-112.
46. Sneeringer CJ, Scott MP, Kuntz KW, et al. Coordinated activities of wild-type plus mutant EZH2 drive tumor-associated hypertrimethylation of lysine 27 on histone H3 (H3K27) in human B-cell lymphomas. Proc Natl Acad Sci U S A. 2010;107(49):20980-20985.

47. Ferry L, Fournier A, Tsusaka T, et al. Methylation of DNA ligase 1 by G9a/GLP recruits UHRF1 to replicating DNA and regulates DNA methylation. Mol Cell. 2017;67(4):550-565.

48. Nakamura K, Baba Y, Kosumi K, et al. UHRF1 regulates global DNA hypomethylation and is associated with poor prognosis in esophageal squamous cell carcinoma. Oncotarget. 2016;7(36):57821-57831.

49. Jia Y, Li P, Fang L, et al. Negative regulation of DNMT3A de novo DNA methylation by frequently overexpressed UHRF family proteins as a mechanism for widespread DNA hypomethylation in cancer. Cell Discov. 2016;2:16007.

50. Hong JH, Jin EH, Kim S, Song KS, Sung JK. LINE-1 hypomethylation is inversely correlated with UHRF1 overexpression in gastric cancer. Oncol Lett. 2018;15(5):6666-6670.

51. Cordaux R, Batzer MA. The impact of retrotransposons on human genome evolution. Nat Rev Genet. 2009;10(10):691-703.

52. Ye J, Zhang Y, Liang W, Huang J, Wang L, Zhong X. UHRF1 is an independent prognostic factor and a potential therapeutic target of esophageal squamous cell carcinoma. J Cancer. 2017;8(19): 4027-4039.

53. Hoshimoto S, Takeuchi H, Ono S, et al. Genome-wide hypomethylation and specific tumor-related gene hypermethylation are associated with esophageal squamous cell carcinoma outcome. J Thorac Oncol. 2015; 10(3):509-517.

54. Jeltsch A. On the enzymatic properties of Dnmt1: specificity, processivity, mechanism of linear diffusion and allosteric regulation of the enzyme. Epigenetics. 2006;1(2):63-66.

55. Arand J, Spieler D, Karius T, et al. In vivo control of CpG and non-CpG DNA methylation by DNA methyltransferases. PLoS Genetics. 2012; 8(6):e1002750.

56. Li Y, Zhang Z, Chen J, et al. Stella safeguards the oocyte methylome by preventing de novo methylation mediated by DNMT1. Nature. 2018; 564(7734):136-140.

57. Bronner C, Chataigneau T, Schini-Kerth VB, Landry Y. The "Epigenetic Code Replication Machinery", ECREM: a promising drugable target of the epigenetic cell memory. Curr Med Chem. 2007;14(25):2629-2641.

58. Chuang LS, Ian HI, Koh TW, Ng HH, Xu G, Li BF. Human DNA(cytosine-5) methyltransferase-PCNA complex as a target for p21WAF1. Science. 1997;277(5334):1996-2000.

59. Schermelleh L, Haemmer A, Spada F, et al. Dynamics of Dnmt1 interaction with the replication machinery and its role in postreplicative maintenance of DNA methylation. Nucleic Acids Res. 2007;35(13):4301-4312.

60. Cartron PF, Blanquart C, Hervouet E, Gregoire M, Vallette FM. HDAC1-mSin3a-NCOR1, Dnmt3b-HDAC1-Egr1 and Dnmt1-PCNAUHRF1-G9a regulate the NY-ESO1 gene expression. Mol Oncol. 2013; $7(3): 452-463$

61. Dai C, Shi D, Gu W. Negative regulation of the acetyltransferase TIP60p53 interplay by UHRF1 (ubiquitin-like with PHD and RING finger domains 1). J Biol Chem. 2013;288(27):19581-19592.

62. Ashraf W, Bronner C, Zaayter L, et al. Interaction of the epigenetic integrator UHRF1 with the MYST domain of TIP60 inside the cell. $J$ Exp Clin Cancer Res. 2017;36(1):188.

63. Achour M, Fuhrmann G, Alhosin M, et al. UHRF1 recruits the histone acetyltransferase Tip60 and controls its expression and activity. Biochem Biophys Res Commun. 2009;390(3):523-528.

64. du Z, Song J, Wang Y, et al. DNMT1 stability is regulated by proteins coordinating deubiquitination and acetylation-driven ubiquitination. Sci Signal. 2010;3(146):ra80.

65. de Marzo AM, Marchi VL, Yang ES, Veeraswamy R, Lin X, Nelson WG. Abnormal regulation of DNA methyltransferase expression during colorectal carcinogenesis. Cancer Res. 1999;59(16):3855-3860.

66. Agoston AT, Argani P, Yegnasubramanian S, et al. Increased protein stability causes DNA methyltransferase 1 dysregulation in breast cancer. J Biol Chem. 2005;280(18):18302-18310. 
67. Jung HJ, Byun HO, Jee BA, et al. The ubiquitin-like with PHD and ring finger domains 1 (UHRF1)/DNA methyltransferase 1 (DNMT1) axis is a primary regulator of cell senescence. J Biol Chem. 2017;292(9): 3729-3739.

68. Seo JS, Choi YH, Moon JW, Kim HS, Park SH. Hinokitiol induces DNA demethylation via DNMT1 and UHRF1 inhibition in colon cancer cells. BMC Cell Biol. 2017;18(1):14.

69. Krifa M, Leloup L, Ghedira K, Mousli M, Chekir-Ghedira L. Luteolin induces apoptosis in BE colorectal cancer cells by downregulating calpain, UHRF1, and DNMT1 expressions. Nutr Cancer. 2014;66(7): $1220-1227$.

70. Shamma A, Suzuki M, Hayashi N, et al. ATM mediates pRB function to control DNMT1 protein stability and DNA methylation. Mol Cell Biol. 2013;33(16):3113-3124.

71. $\mathrm{Ma} \mathrm{H}$, Chen $\mathrm{H}$, Guo X, et al. M phase phosphorylation of the epigenetic regulator UHRF1 regulates its physical association with the deubiquitylase USP7 and stability. Proc Natl Acad Sci U S A. 2012;109(13): 4828-4833.

72. Zhang ZM, Rothbart SB, Allison DF, et al. An allosteric interaction links USP7 to deubiquitination and chromatin targeting of UHRF1. Cell Rep. 2015;12(9):1400-1406.

73. Chu J, Loughlin EA, Gaur NA, et al. UHRF1 phosphorylation by cyclin A2/cyclin-dependent kinase 2 is required for zebrafish embryogenesis. Mol Biol Cell. 2012;23(1):59-70.

74. Dar A, Shibata E, Dutta A. Deubiquitination of Tip60 by USP7 determines the activity of the p53-dependent apoptotic pathway. Mol Cell Biol. 2013;33(16):3309-3320.

75. Yamaguchi L, Nishiyama A, Misaki T, et al. Usp7-dependent histone H3 deubiquitylation regulates maintenance of DNA methylation. Sci Rep 2017;7(1):55.

76. Cheng J, Yang H, Fang J, et al. Molecular mechanism for USP7-mediated DNMT1 stabilization by acetylation. Nat Commun. 2015;6:7023.

77. Cedar H, Bergman Y. Linking DNA methylation and histone modification: patterns and paradigms. Nat Rev Genet. 2009;10(5):295-304.

78. Zhou Q, Agoston AT, Atadja P, Nelson WG, Davidson NE. Inhibition of histone deacetylases promotes ubiquitin-dependent proteasomal degradation of DNA methyltransferase 1 in human breast cancer cells. Mol Cancer Res. 2008;6(5):873-883.

79. Kim JK, Estève PO, Jacobsen SE, Pradhan S. UHRF1 binds G9a and participates in p21 transcriptional regulation in mammalian cells. Nucleic Acids Res. 2009;37(2):493-505.

80. Lachner M, O'Carroll D, Rea S, Mechtler K, Jenuwein T. Methylation of histone $\mathrm{H} 3$ lysine 9 creates a binding site for HP1 proteins. Nature. 2001;410(6824):116-120.

81. Tachibana M, Sugimoto K, Fukushima T, Shinkai Y. Set domaincontaining protein, G9a, is a novel lysine-preferring mammalian histone methyltransferase with hyperactivity and specific selectivity to lysines 9 and 27 of histone H3. J Biol Chem. 2001;276(27):25309-25317.

82. Ikegami K, Iwatani M, Suzuki M, et al. Genome-wide and locus-specific DNA hypomethylation in G9a deficient mouse embryonic stem cells. Genes Cells. 2007;12(1):1-11.

83. Dong KB, Maksakova IA, Mohn F, et al. DNA methylation in ES cells requires the lysine methyltransferase G9a but not its catalytic activity. EMBO J. 2008;27(20):2691-2701.

84. Leung DC, Dong KB, Maksakova IA, et al. Lysine methyltransferase G9a is required for de novo DNA methylation and the establishment, but not the maintenance, of proviral silencing. Proc Natl Acad Sci USA. 2011;108(14):5718-5723.

85. Zhou L, Shang Y, Jin Z, et al. UHRF1 promotes proliferation of gastric cancer via mediating tumor suppressor gene hypermethylation. Cancer Biol Ther. 2015;16(8):1241-1251.

86. Zhu M, Xu Y, Ge M, Gui Z, Yan F. Regulation of UHRF1 by microRNA-9 modulates colorectal cancer cell proliferation and apoptosis. Cancer Sci. 2015;106(7):833-839.

87. Mudbhary R, Hoshida Y, Chernyavskaya Y, et al. UHRF1 overexpression drives DNA hypomethylation and hepatocellular carcinoma. Cancer Cell. 2014;25(2):196-209.
88. Yang $\mathrm{C}$, Wang $\mathrm{Y}$, Zhang $\mathrm{F}$, et al. Inhibiting UHRF1 expression enhances radiosensitivity in human esophageal squamous cell carcinoma. Mol Biol Rep. 2013;40(9):5225-5235.

89. Du S, Xu G, Zou W, Xiang T, Luo Z. Effect of dihydroartemisinin on UHRF1 gene expression in human prostate cancer PC-3 cells. Anticancer Drugs. 2017;28(4):384-391.

90. Jazirehi AR, Arle D, Wenn PB. UHRF1: a master regulator in prostate cancer. Epigenomics. 2012;4(3):251-252.

91. Saidi S, Popov Z, Janevska V, Panov S. Overexpression of UHRF1 gene correlates with the major clinicopathological parameters in urinary bladder cancer. Int Braz J Urol. 2017;43(2):224-229.

92. Fang L, Shanqu L, Ping G, et al. Gene therapy with RNAi targeting UHRF1 driven by tumor-specific promoter inhibits tumor growth and enhances the sensitivity of chemotherapeutic drug in breast cancer in vitro and in vivo. Cancer Chemother Pharmacol. 2012;69(4):1079-1087.

93. Unoki M, Daigo Y, Koinuma J, Tsuchiya E, Hamamoto R, Nakamura Y. UHRF1 is a novel diagnostic marker of lung cancer. Br J Cancer. 2010;103(2):217-222.

94. Daskalos A, Oleksiewicz U, Filia A, et al. UHRF1-mediated tumor suppressor gene inactivation in nonsmall cell lung cancer. Cancer. 2011;117(5):1027-1037.

95. Abusnina A, Keravis T, Yougbaré I, Bronner C, Lugnier C. Antiproliferative effect of curcumin on melanoma cells is mediated by PDE1A inhibition that regulates the epigenetic integrator UHRF1. Mol Nutr Food Res. 2011;55(11):1677-1689.

96. Zhang ZY, Zhu B, Zhao XW, et al. Regulation of UHRF1 by microRNA-378 modulates medulloblastoma cell proliferation and apoptosis. Oncol Rep. 2017;38(5):3078-3084.

97. Alhosin M, Abusnina A, Achour M, et al. Induction of apoptosis by thymoquinone in lymphoblastic leukemia Jurkat cells is mediated by a p73-dependent pathway which targets the epigenetic integrator UHRF1. Biochem Pharmacol. 2010;79(9):1251-1260.

98. Liu W, Qiao RH, Wang DM, Huang XW, Li B, Wang D. UHRF1 promotes human osteosarcoma cell invasion by downregulating the expression of E-cadherin in an Rb1-dependent manner. Mol Med Rep. 2016;13(1):315-320.

99. Boukhari A, Alhosin M, Bronner C, et al. CD47 activation-induced UHRF1 over-expression is associated with silencing of tumor suppressor gene p16INK4A in glioblastoma cells. Anticancer Res. 2015;35(1):149-157.

100. Wang F, Yang YZ, Shi CZ, et al. UHRF1 promotes cell growth and metastasis through repression of p16(ink4a) in colorectal cancer. Ann Surg Oncol. 2012;19(8):2753-2762.

101. Krifa M, Alhosin M, Muller CD, et al. Limoniastrum guyonianum aqueous gall extract induces apoptosis in human cervical cancer cells involving p16INK4A re-expression related to UHRF1 and DNMT1 down-regulation. $J$ Exp Clin Cancer Res. 2013;32(1):30.

102. Jin W, Chen L, Chen Y, et al. UHRF1 is associated with epigenetic silencing of BRCA1 in sporadic breast cancer. Breast Cancer Res Treat. 2010;123(2):359-373.

103. Zhang Y, Huang Z, Zhu Z, et al. Upregulated UHRF1 promotes bladder cancer cell invasion by epigenetic silencing of KiSS1. PLoS One. 2014;9(10):e104252.

104. Zhuo H, Tang J, Lin Z, et al. The aberrant expression of MEG3 regulated by UHRF1 predicts the prognosis of hepatocellular carcinoma. Mol Carcinog. 2016;55(2):209-219.

105. Zhou L, Zhao X, Han Y, et al. Regulation of UHRF1 by miR-146a/b modulates gastric cancer invasion and metastasis. FASEB J. 2013; 27(12):4929-4939.

106. Abu-Alainin W, Gana T, Liloglou T, et al. UHRF1 regulation of the Keap1-Nrf2 pathway in pancreatic cancer contributes to oncogenesis. $J$ Pathol. 2016;238(3):423-433.

107. Gao SP, Sun HF, Li LD, Fu WY, Jin W. UHRF1 promotes breast cancer progression by suppressing KLF17 expression by hypermethylating its promoter. Am J Cancer Res. 2017;7(7):1554-1565.

108. Yang C, Li J, Yu L, et al. Regulation of RIP3 by the transcription factor $\mathrm{Sp} 1$ and the epigenetic regulator UHRF1 modulates cancer cell necroptosis. Cell Death Dis. 2017;8(10):e3084. 
109. Sheng Y, Wang H, Liu D, et al. Methylation of tumor suppressor gene CDH13 and SHP1 promoters and their epigenetic regulation by the UHRF1/PRMT5 complex in endometrial carcinoma. Gynecol Oncol. 2016;140(1):145-151.

110. Sabatino L, Fucci A, Pancione M, et al. UHRF1 coordinates peroxisome proliferator activated receptor gamma (PPARG) epigenetic silencing and mediates colorectal cancer progression. Oncogene. 2012; 31(49):5061-5072.

111. Magnani E, Macchi F, Mancini M. UHRF1 regulates CDH1 via promoter associated non-coding RNAs in prostate cancer cells. Biochimica et biophysica acta. 2018;1861:258-270.

112. Beck A, Trippel F, Wagner A, et al. Overexpression of UHRF1 promotes silencing of tumor suppressor genes and predicts outcome in hepatoblastoma. Clin Epigenetics. 2018;10:27.

113. Ying L, Lin J, Qiu F, et al. Epigenetic repression of regulator of G-protein signaling 2 by ubiquitin-like with PHD and ring-finger domain 1 promotes bladder cancer progression. FEBS J. 2015;282(1):174-182.

114. Zhang Q, Qiao L, Wang X, Ding C, Chen JJ. UHRF1 epigenetically down-regulates $\mathrm{UbcH} 8$ to inhibit apoptosis in cervical cancer cells. Cell Cycle. 2018;17(3):300-308.

115. Ye Z, Shen N, Weng Y, et al. Low miR-145 silenced by DNA methylation promotes NSCLC cell proliferation, migration and invasion by targeting mucin 1. Cancer Biol Ther. 2015;16(7):1071-1079.

116. Unoki M, Nishidate T, Nakamura Y. ICBP90, an E2F-1 target, recruits HDAC1 and binds to methyl-CpG through its SRA domain. Oncogene. 2004;23(46):7601-7610.

117. Park S-A, Platt J, Lee JW, López-Giráldez F, Herbst RS, Koo JS. E2F8 as a Novel Therapeutic Target for Lung Cancer. J Natl Cancer Inst. 2015;107:djv151.

118. Sanders DA, Gormally MV, Marsico G, Beraldi D, Tannahill D, Balasubramanian S. FOXM1 binds directly to non-consensus sequences in the human genome. Genome Biol. 2015;16:130.

119. Wu SM, Cheng WL, Liao CJ, et al. Negative modulation of the epigenetic regulator, UHRF1, by thyroid hormone receptors suppresses liver cancer cell growth. Int J Cancer. 2015;137(1):37-49.

120. Wang J, Cheng Y, Yin X, et al. Globular adiponectin inhibits leptinstimulated esophageal adenocarcinoma cell proliferation via adiponectin receptor 2-mediated suppression of UHRF1. Mol Cell Biochem. 2017;431(1-2):103-112.

121. Ding G, Chen P, Zhang H, et al. Regulation of ubiquitin-like with plant homeodomain and RING finger domain 1 (UHRF1) protein stability by heat shock protein 90 chaperone machinery. J Biol Chem. 2016;291(38):20125-20135

122. Arima Y, Hirota T, Bronner C, et al. Down-regulation of nuclear protein ICBP90 by p53/p21Cip1/WAF1-dependent DNA-damage checkpoint signals contributes to cell cycle arrest at G1/S transition. Genes Cells. 2004;9(2):131-142.

123. Chen J, Sheng X, Ma H, et al. WDR79 mediates the proliferation of non-small cell lung cancer cells by regulating the stability of UHRF1. J Cell Mol Med. 2018;22(5):2856-2864.

124. Wang X, Wu Q, Xu B, et al. miR-124 exerts tumor suppressive functions on the cell proliferation, motility and angiogenesis of bladder cancer by fine-tuning UHRF1. FEBS J. 2015;282(22):4376-4388.

125. Goto Y, Kurozumi A, Nohata N, et al. The microRNA signature of patients with sunitinib failure: regulation of UHRF1 pathways by microRNA-101 in renal cell carcinoma. Oncotarget. 2016;7(37):59070-59086.

126. Deng W, Yan M, Yu T, et al. Quantitative proteomic analysis of the metastasis-inhibitory mechanism of miR-193a-3p in non-small cell lung cancer. Cell Physiol Biochem. 2015;35(5):1677-1688.

127. Atala A. Re: Regulation of UHRF1 by dual-strand tumor-suppressor microRNA-145 (miR-145-5p and miR-145-3p): inhibition of bladder cancer cell aggressiveness. J Urol. 2016;196(4):1314.

128. Peng R, Liu H, Peng H, et al. Promoter hypermethylation of let-7a-3 is relevant to its down-expression in diabetic nephropathy by targeting UHRF1. Gene. 2015;570(1):57-63.
129. Chen $\mathrm{H}, \mathrm{Ma} H$, Inuzuka $H$, et al. DNA damage regulates UHRF1 stability via the $\mathrm{SCF}^{(\beta-T r C P)} \mathrm{E} 3$ ligase. Mol Cell Biol. 2013;33(6):1139-1148.

130. Yang J, Liu K, Yang J, et al. PIM1 induces cellular senescence through phosphorylation of UHRF1 at Ser311. Oncogene. 2017; 36(34):4828-4842.

131. Veland N, Hardikar S, Zhong Y, et al. The arginine methyltransferase PRMT6 regulates DNA methylation and contributes to global DNA hypomethylation in cancer. Cell Rep. 2017;21(12):3390-3397.

132. Graf U, Casanova EA, Wyck S, et al. Pramel7 mediates ground-state pluripotency through proteasomal-epigenetic combined pathways. Nat Cell Biol. 2017;19(7):763-773.

133. Myrianthopoulos V, Cartron PF, Liutkevičiūtė Z, et al. Tandem virtual screening targeting the SRA domain of UHRF1 identifies a novel chemical tool modulating DNA methylation. Eur J Med Chem. 2016; 114:390-396.

134. Jang SY, Hong D, Jeong SY, Kim JH. Shikonin causes apoptosis by up-regulating p73 and down-regulating ICBP90 in human cancer cells. Biochem Biophys Res Commun. 2015;465(1):71-76.

135. Achour M, Mousli M, Alhosin M, et al. Epigallocatechin-3-gallate up-regulates tumor suppressor gene expression via a reactive oxygen species-dependent down-regulation of UHRF1. Biochem Biophys Res Commun. 2013;430(1):208-212.

136. Lin Y, Chen W, Wang Z, Cai P. Emodin promotes the arrest of human lymphoma Raji cell proliferation through the UHRF1-DNMT3A- $\triangle$ Np73 pathways. Mol Med Rep. 2017;16(5):6544-6551.

137. Wang C, Wang X, Su Z, Fei H, Liu X, Pan Q. The novel mTOR inhibitor Torin-2 induces autophagy and downregulates the expression of UHRF1 to suppress hepatocarcinoma cell growth. Oncol Rep. 2015; 34(4):1708-1716.

138. Fang Z, Xing F, Bronner C, Teng Z, Guo Z. ICBP90 mediates the ERK1/2 signaling to regulate the proliferation of Jurkat T cells. Cell Immunol. 2009;257(1-2):80-87.

139. Yu C, Xing F, Tang Z, et al. Anisomycin suppresses Jurkat T cell growth by the cell cycle-regulating proteins. Pharmacol Rep. 2013; 65(2):435-444.

140. Parashar G, Capalash N. Promoter methylation-independent reactivation of PAX1 by curcumin and resveratrol is mediated by UHRF1. Clin Exp Med. 2016;16(3):471-478.

141. Tien AL, Senbanerjee S, Kulkarni A, et al. UHRF1 depletion causes a G2/M arrest, activation of DNA damage response and apoptosis. Biochem J. 2011;435(1):175-185.

142. Li X, Meng Q, Rosen EM, Fan S. UHRF1 confers radioresistance to human breast cancer cells. Int J Radiat Biol. 2011;87(3):263-273.

143. Ge TT, Yang M, Chen Z, Lou G, Gu T. UHRF1 gene silencing inhibits cell proliferation and promotes cell apoptosis in human cervical squamous cell carcinoma CaSki cells. J Ovarian Res. 2016;9(1):42.

144. Maenohara S, Unoki M, Toh H, et al. Role of UHRF1 in de novo DNA methylation in oocytes and maintenance methylation in preimplantation embryos. PLoS Genet. 2017;13(10):e1007042.

145. Cui Y, Chen X, Zhang J, et al. Uhrf1 controls iNKT cell survival and differentiation through the Akt-mTOR axis. Cell Rep. 2016;15(2): 256-263

146. Obata Y, Furusawa Y, Endo TA, et al. The epigenetic regulator Uhrf1 facilitates the proliferation and maturation of colonic regulatory T cells. Nat Immunol. 2014;15(6):571-579.

147. Elia L, Kunderfranco P, Carullo P, et al. UHRF1 epigenetically orchestrates smooth muscle cell plasticity in arterial disease. J Clin Invest. 2018;128(6):2473-2486.

148. Pan FP, Zhou HK, Bu HQ, et al. Emodin enhances the demethylation by 5-Aza-CdR of pancreatic cancer cell tumor-suppressor genes P16, RASSF1A and ppENK. Oncol Rep. 2016;35(4):1941-1949.

149. Ehrlich M, Gama-Sosa MA, Huang LH, et al. Amount and distribution of 5-methylcytosine in human DNA from different types of tissues of cells. Nucleic Acids Res. 1982;10(8):2709-2721. 
OncoTargets and Therapy

\section{Publish your work in this journal}

OncoTargets and Therapy is an international, peer-reviewed, open access journal focusing on the pathological basis of all cancers, potential targets for therapy and treatment protocols employed to improve the management of cancer patients. The journal also focuses on the impact of management programs and new therapeutic agents and protocols on

patient perspectives such as quality of life, adherence and satisfaction. The manuscript management system is completely online and includes a very quick and fair peer-review system, which is all easy to use. Visit http://www.dovepress.com/testimonials.php to read real quotes from published authors.

Submit your manuscript here: http://www.dovepress.com/oncotargets-and-therapy-journal 